\title{
Effect of Loureirin B on Crohn's disease rat model induced by TNBS via IL-6/STAT3/NF-KB signaling pathway
}

\author{
Xueliang Sun ${ }^{\dagger}, K$ Wen ${ }^{\dagger}$, Zhizhong Xu, Zongqi He, Bensheng Wu, Xiao Yang and Xiaopeng Wang ${ }^{*}$
}

\begin{abstract}
Background: Crohn's disease (CD) is a chronic relapsing form of inflammatory bowel disease, seriously threatening human beings health. However, the pathogenesis of CD is still unclear and there is no specific effective drug for treatment of CD. Resina Donis (RD) obtained from Dracaena cochinchinensis (Lour.) S. C. Chen (Liliaceae), has been used for the treatment of CD clinically. Loureirin B $(\mathrm{LB})$ is one of the most important chemical compositions and physiologically active ingredients of resina draconis. It has the molecular structure propan-1-one, 1-(4-hydroxyphenyl)3-(2,4,6-trimethoxyphenyl)-1-(4-hydroxyphenyl)-3-(2,4,6-trimethoxyphenyl) propan-1-one. The aim of this study was to investigate the effect of $L B$ on $C D$ and explore the underlying mechanisms.

Methods and results: In this study, the result demonstrated that LB prolonged the survival time of 2,4,6-trinitrobenzene sulfonic acid (TNBS)-induced rats and alleviated colonic damage in a dose dependent manner. Besides, LB remarkably ameliorated TNBS-induced inflammatory response via regulation of cytokines in the colonic tissues. Moreover, LB could reverse the established fibrosis and impede the accumulation infiltration, and improve the apoptosis induced by TNBS in a dose dependent manner. Further, LB dramatically suppressed TNBS-induced the activation of IL-6/STAT3/NF-KB signaling pathway.
\end{abstract}

Conclusions: These findings suggested that LB could be beneficial regarding ameliorating TNBS-induced CD, which may represent a novel approach to treat $C D$ and provide an alternative choice for disorders associated with CD.

Keywords: CD, LB, Inflammation, Fibrosis, IL-6/STAT3/NF-KB

\section{Background}

$\mathrm{CD}$ is a chronic relapsing form of inflammatory bowel disease, typically characterized with transmural inflammation, lymphangiectasia, and lymphatic and fibrous tissue hyperplasia [1,2]. CD can be involved in all segments of the digestive tract, and bring about perforation and abdominal adenoma and other complications, which seriously affects the life quality of patients [3]. Thus, CD is a difficult disease urgently needed to be solved. CD occurs

*Correspondence: wxpeng2004@163.com

tXueliang Sun and Ke Wen contributed equally to this work Department of Colorectal Surgery, Suzhou TCM Hospital Affiliated

to Nanjing University of Chinese Medicine, Suzhou 215000, Jiangsu, China mostly in developed countries such as Europe and America, the incidence of CD is 43.6 per 100,000 in Europeans and Americans, and 5.6 per 10 million in Asians [4]. In recent years, with the changes of living conditions and the continuous improvement of clinical diagnosis level, the incidence of $\mathrm{CD}$ in China has been increasing year by year $[5,6]$. Compared with the clinical diagnosis of $\mathrm{CD}$, the research progress of the treatment for $C D$ is relatively slow. In addition, the pathogenesis of $C D$ is still unclear, and $\mathrm{CD}$ is thought to be the results of heredity, immune disorders, intestinal barrier dysfunction and intestinal microbial interaction [7]. It is precisely because the current research can not reveal the exact pathogenesis of 
$\mathrm{CD}$, so there are no specific effective treatment methods or drugs for CD [8].

$\mathrm{CD}$ is mainly characterized by nonspecific inflammation of the digestive tract, which occurs in the individual intestinal tract with genetic susceptibility and does not depend on specific pathogens [9]. Studies have shown that lamina propria $\mathrm{T}$ cells play a key role in the induction and persistence of intestinal inflammation. Interaction between antigen presenting cells in intestinal mucosal immune system and local bacterial flora leads to the uncontrolled activation of mucosal $\mathrm{CD} 4^{+} \mathrm{T}$ cells, and sustained release of inflammatory factors such as tumor necrosis factor- $\alpha$ (TNF- $\alpha$ ), interleukin-6 (IL-6), IL-12, IL-23, IL-27 and IL-17, which are important factors resulting in intestinal injury in CD patients $[10,11]$. IL-6 can be produced by multiple cells and has pleiotropic effects on different organ systems. In the pathological state of $\mathrm{CD}, \mathrm{CD}^{+}{ }^{+} \mathrm{T}$ cells exhibit apoptosis resistance, and the accumulation of $\mathrm{CD} 4^{+} \mathrm{T}$ cells in inflammatory sites mainly depends on anti-apoptotic IL- 6 transmembrane signaling pathway [12]. IL-6 trans-membrane signaling pathway can induce the activation of STAT3, and STAT3 itself can further induce the expression of anti-apoptotic factors $\mathrm{Bcl}-2$ and Bax-xl, causing $\mathrm{T}$ cells resistance to apoptosis $[13,14]$. Besides, $T$ cell apoptosis resistance and aggregation eventually lead to a vicious cycle of chronic inflammation, which can be effectively blocked by anti-IL- 6 receptor antibody, indicating that IL-6/STAT3 signaling pathway plays a central role in apoptosis resistance in the pathological state of $\mathrm{CD}$ [15]. In addition, recent studies have found that IL- 6 signaling pathway plays an important role in the occurrence and differentiation of Th17, suggesting that IL-6/STAT3 trans-membrane signaling pathway is essential for $\mathrm{T}$ cells apoptosis resistance and type Th17 immune reaction $[16,17]$. Further, in inflammatory cells, the inappropriate activation of nuclear factor- $\mathrm{\kappa B}$ (NF- $\mathrm{\kappa B}$ ) is the key transcription factor that regulates the expression of the inflammatory mediators, which is related to the occurrence and development of CD inflammation [18].

RD, obtained from Dracaena cochinchinensis (Lour.) $S$. C. Chen (Liliaceae), is a Chinese herb with the positive activities of promoting blood circulation for removing blood stasis, regenerating tissue to heal wound, relieving pain and eliminating swelling, which has been commonly used for the treatment of coronary heart disease, angina, and acute myocardial infarction [19,20]. Additionally, the ethyl acetate of RD can promote inflammatory response induced by LPS through inhibiting ROS production in vascular smooth muscle cells and macrophages [21]. Moreover, the clinical effect of RD on CD is satisfactory in China [22]. LB is one of the most important chemical compositions and physiologically active ingredients of resina draconis. It has the molecular structure propan1-one, 1-(4-hydroxyphenyl)-3-(2,4,6-trimethoxyphenyl)1-(4-hydroxyphenyl)-3-(2,4,6-trimethoxyphenyl) propan-1-one. The previous study reported that LB could inhibit the hepatic stellate cell proliferation by regulating miR-148-3p via Wnt/ $\beta$-catenin signaling pathway [23]. LB, an essential component of Sanguis Draxonis, inhibits Kv1.3 channel and suppresses cytokine release from Jurkat $\mathrm{T}$ cells [24]. LB inhibited fibroblast proliferation and extracellular matrix deposition in hypertrophic scar via TGF- $\beta$ /Smad pathway [25]. However, the role of LB on $\mathrm{CD}$ and the underlying mechanisms remain unknown. Therefore, in the current study, we investigated the effect of LB on CD rat model induced by TNBS and explored the possible mechanisms.

\section{Methods}

\section{Experimental materials}

Sixty Sprague-Dawley (SD) rats (equal ratio of male and female), weighing $250 \pm 20$ g, were obtained from Experimental Animal Center of Nanjing University (Nanjing, China). Sulphasalazine (SASP) $(250 \mathrm{mg}$ ) was purchased from the National Institutes for Food and Drug Control (Beijing, China). Trinitro-benzene-sulfonic acid (TNBS) was purchased from Sigma Chemical (St. Louis, MO, USA).

\section{Preparation of LB}

LB in the present study was followed as previous recommendations [26]. LB was obtained from the National Institute for the Control of Pharmaceutical and Biological Products of China and reconstituted in DMSO at a final stock concentration of $25 \mathrm{mg} / \mathrm{mL}$.

\section{Establishment of the $C D$ rat model}

The CD rat model was induced using TNBS as described as previous [27]. In brief, SD rats were fasted overnight and lightly anesthetized with ether. Then, TNBS (5 mg), dissolved in $0.2 \mathrm{~mL}$ of $50 \%$ ethanol, was injected into the descending colon, and the rats from the control group were only treated with $0.2 \mathrm{~mL}$ of $50 \%$ ethanol following the same method once a week for a total of four treatments.

\section{Experimental animals grouping and treatments}

CD rats were randomly divided into six groups $(n=10)$, including the control group, the model group, the SASP group, and the LB groups (25, 50 and $100 \mathrm{mg} / \mathrm{kg}$ ). In the control group, rats were only administered with normal saline via oral gavage every day. The rats from the model group were treated with TNBS once a week for a total of four treatments and administrated with normal saline via 
oral gavage every day. The rats of the SASP group were treated with prepared SASP suspension liquid $(0.1 \mathrm{~g} / \mathrm{mL})$ daily via oral gavage based on the model group, SASP was an effective drug for CD $[28,29]$. The LB group rats were administered with 25,50 and $100 \mathrm{mg} / \mathrm{kg} \mathrm{LB}$ every day for a total of $28 \mathrm{~d}$ via oral gavage based on the model group.

\section{Assessment of colonic damage}

The disease activity index (DAI) of all the rats from different groups was evaluated daily according to criteria [30, 31] (Table 1). Every group rats were checked the weight and euthanized at day 28 . Then the distal colon was carefully excised and the colon was weighed and measured length.

\section{Hematoxylin and eosin (H\&E) staining}

Colonic segments were excised and washed with phosphate buffered saline (PBS). Then the sections were dehydrated by alcohol, cleared in xylene and embedded in paraffin. The colonic sections of $5 \mu \mathrm{m}$ thickness were transversely cut and stained with $\mathrm{H} \& \mathrm{E}$ for visual analysis.

\section{Masson staining assay}

Isolated colonic tissues were fixed in $4 \%$ neutral formalin and embedded in paraffin. Then the sections $(5 \mu \mathrm{m})$ were stained with Masson trichrome solutions. Images were obtained using a light microscope.

\section{TUNEL assay}

Apoptosis was analyzed using a terminal deoxynucleotidyl transferase-mediated dUTP-biotin nick end labeling (TUNEL) assay kit following the manufacturer's instructions. In brief, the sections $(5 \mu \mathrm{m})$ of isolated colonic tissues were incubated with fluorescein isothiocyanate (FITC)-labeled dUTP and terminal deoxynucleotidyl transferase for $1 \mathrm{~h}$ at $37^{\circ} \mathrm{C}$. Nuclei counterstaining was performed using DAPI, and examined under a fluorescence microscope (Olympus, Tokyo, Japan).

Table 1 Criteria for assessment of colonic macroscopic and microscopic damage

\begin{tabular}{llll}
\hline Score & Body weight loss & Stool character & Fecal occult blood \\
\hline 0 & 0 & Normal formed & Negative \\
1 & $=1$ to $5 \%$ & & \\
2 & $=5$ to $10 \%$ & Loose stool & Positive \\
3 & $=10$ to $20 \%$ & & \\
4 & $>20 \%$ & Diarrhea & Gross bleeding \\
\hline
\end{tabular}

\section{Immunohistochemical analysis}

Isolated colonic tissues from different groups were immunohistochemically stained for Slug (ab27568; Abcam; 1:1000 dilution), Snail (ab53519; Abcam; 1:500 dilution), N-cadherin (ab18203; Abcam; 1:300 dilution), E-cadherin (ab1416; Abcam; 1:100 dilution), Vimentin (ab8978; Abcam; 1:200 dilution), Bax (ab32503; Abcam; 1:250 dilution), Bcl-2 (ab32124; Abcam; 1:500 dilution), Caspase-3 (ab13847; Abcam; 1:500 dilution), Caspase-9 (ab32539; Abcam; 1:250 dilution). Briefly, isolated colonic tissues were fixed in $4 \%$ neutral formalin for $24 \mathrm{~h}$, embedded in paraffin and were serially sectioned at $5 \mu \mathrm{m}$. Sections were deparaffinized and rehydrated, then submerged in hydrogen peroxide to quench peroxidase activity following incubated with $1 \%$ BSA to block nonspecific binding sites. After incubation with indicated primary antibodies at $4{ }^{\circ} \mathrm{C}$ for $12 \mathrm{~h}$, secondary antibodies IgG (ab6721; Abcam; 1:1000 dilution) were applied to slides for $1 \mathrm{~h}$ at room temperature. For immunohistochemical analysis, the slice was colored by diaminobenzidine kit (DAB; Beyotime) followed by counterstaining with hematoxylin. All the sections were visualized using diaminobenzidine (DAB, Beyotime) under a light microscope (Nikon 80i).

\section{Enzyme-linked immunosorbent assay (ELISA)}

The concentrations of cytokines in isolated colonic tissues were determined by enzyme-linked immunosorbent assay (ELISA) for rat IL-1, IL-1 $\beta$, IL-6, IL-10, TNF- $\alpha$, TGF- $\beta$, INOS, MPO, SIgA and MDA (eBioscience, San Diego, $C A)$ following the manufacturer's instructions.

\section{Quantitative real-time PCR assay (qRT-PCR)}

Total RNA of isolated colonic tissues was extracted with Trizol reagent (Takara, China) and was reverse-transcribed into cDNA using a PrimeScript RT reagent kit (Takara, China) according to the manufacturer's instructions. Quantitative PCR (ABI 7500, USA) was performed using SYBR green kit (Takara, China) to detect the relative mRNA expression levels of IL-6, p-STAT3, STAT3, $\mathrm{p}-\mathrm{NF}-\mathrm{kB}$ (p65) and NF-kB (p65). GAPDH was normalized to an endogenous reference. The relative gene expression level was calculated by using the comparative $\mathrm{Ct}$ method, and those relative to the calibrator were given by the formula $2^{-\Delta \Delta \mathrm{Ct}}$. The PCR primers for IL- 6 were 5'-GAGAAAAGAGTTGTGCAATGGC-3' (forward) and $5^{\prime}$-ACTAGGTTTGCCGAGTAGAC C-3' (reverse), STAT3 were $5^{\prime}$-GAGGAGGCATTCGGAAAGTATT- $3^{\prime}$ (forward) and 5'-CAGGT CGTTGGTGTCACACA-3' (reverse), NF-kB (p65) were 5'-CACCAAAGACCCACC TCACC- $3^{\prime}$ (forward) and 5'-CCGCATTCAAGTATAG TCCC-3' (reverse), GAPDH were 5'-CGCTAACATC 
AAATGGGGTG- $3^{\prime}$ (forward) and $5^{\prime}$-ACAACCTGG TCCTCAGTGTA-3' (reverse).

\section{Western blotting assay}

The total protein from the isolated colonic tissues was extracted using RIPA lysis buffer (Pierce; Rockford, IL, USA) according to the manufacturer's instructions. Protein concentrations were measured with BCA protein assay kit. Equal amounts of protein samples $(50 \mu \mathrm{g})$ were separated on $12 \%$ sodium dodecyl sulfate-polyacrylamide gel electrophoresis (SDS-PAGE) and transferred to a polyvinylidene difluoride membrane (PVDF) (Millipore; Billerica, MA, USA). Then the PVDF membrane was blocked with $5 \%$ bovine serum album (Amresco, Solon, $\mathrm{OH}, \mathrm{USA}$ ) for $1 \mathrm{~h}$ and incubated with primary antibodies including Slug (cat. no. ab27568; Abcam), Snail (cat. no. ab53519; Abcam), N-cadherin (cat. no. ab18203; Abcam), E-cadherin (cat. no. ab1416; Abcam), Vimentin (cat. no. ab8978; Abcam), Bax (cat. no. ab32503; Abcam), Bcl-2 (cat. no. ab32124; Abcam), Caspase-3 (cat. no. ab13585; Abcam), Caspase-9 (cat. no. ab32539; Abcam), IL-6 (cat. no. ab6672; Abcam), p65 (cat. no. ab16502; Abcam), IKB- $\alpha$ (cat. no. ab32518; Abcam) and GAPDH (cat. no. ab9485; Abcam) at 1:1000 dilution at $4{ }^{\circ} \mathrm{C}$ overnight. After washing with PBS three times, the membranes were further incubated with horseradish peroxidase-conjugated secondary antibody IgG (cat. no. ab6721; 1:2000; Abcam). The blots were visualized using the ECL-plus detection system (GE Healthcare, Buckingham-shire, UK). The membrane was exposed to X-ray film and then developed. The signal intensity was quantified using Gray-scale analysis software (Image Tool 3.00).

\section{Statistical analysis}

Graphpad Prism 5.0 software was performed to analyze all data. The data were represented as mean \pm SD from at least three independent assays. One-way analysis of variance followed by Tukey was applied to compare difference between multiple groups. Additionally, nonparametric tests were applied for data with non-fulfilled distribution assumptions due to some normalized data. A $\mathrm{p}$-value $<0.05$ was considered statistically significant.

\section{Results}

\section{Effect of $L B$ on the survival rate and body weight in rats} induced with TNBS

Firstly, to evaluate whether LB had a protective effect on the rats induced with TNBS, the survival rate and the body weight of TNBS-induced rats were measured. As shown in Fig. 1a, b, TNBS treatment could dramatically decrease the rat body weight, whereas treatment with LB appeared to increase the body weight in a dose dependent manner. Besides, the results demonstrated that the survival rate of rats from the TNBS was lower compared with that in the control group, while the survival time of rats was prolonged after treatment of LB in a dose dependent manner, and the effect of LB with high concentration was better than SASP treatment (Fig. 1c). Moreover, rats from different groups also had intense inflammatory response characterized by DAI. Saline did not protect against colonic injury. However, treatment with SASP significantly inhibited the colonic injury, whereas administration with LB could protect the rat colon against TNBS-induced injury (Fig. 1d).

Effect of $L B$ on the colonic length in rats induced with TNBS Colon shortening is an indirect marker of inflammation [32]. Compared with the control group, the colonic length in model group was significantly decreased, which demonstrated that $C D$ rats had seriously colon injury. After treatment with different concentrations of LB, no obvious change was viewed in the colonic length and weight (Fig. 2a-c). Moreover, the severity of colonic inflammation was evaluated by $\mathrm{H} \& \mathrm{E}$ staining assay. As shown in Fig. 2d, e, the architecture of colonic tissues from the control group was normal and was devoid of any inflammatory infiltration necrosis and edema. Rats induced with TNBS caused a significant elevation in inflammatory response characterized by thickening of the mucosa, inflammatory cell infiltration with necrotic foci, extensive destruction of mucosal epithelium, loss of mucus-secreting cells, submucosal edema, necrosis and ulcer on the mucosal surface. The colonic tissues from SASP group exhibited a downward trend in the inflammatory infiltration, and rats treated with LB showed remarkable inhibitory effect on TNBS-induced colonic histological damage in a dose dependent manner.

\section{Effect of LB on the production of inflammatory cytokines in rats induced with TNBS}

In order to investigate the effect of LB on the production of inflammatory cytokines in rats induced with TNBS, the colonic tissues were collected. As described in Fig. 3, TNBS could significantly elevate the production of IL- 1 , IL- $1 \beta$, IL- 6 , TNF- $\alpha$ and TGF- $\beta$, and decrease the secretion of IL-10. However, LB treatment could obviously reverse these phenomenons, which was that the administration of LB in rat led to a decrease of the production of IL- 1 , IL- $1 \beta$, IL-6, TNF- $\alpha$, IL- 6 , TGF- $\beta$ and an increase of the production of IL-10. These results indicated that $\mathrm{LB}$ had a positive role in the modulation of cytokine production in $\mathrm{CD}$ rats induced with TNBS. 


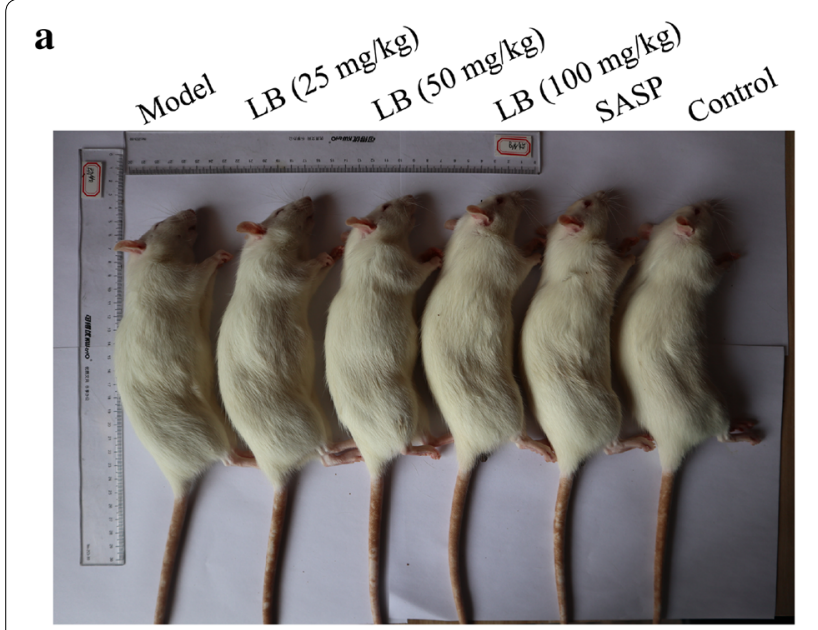

b
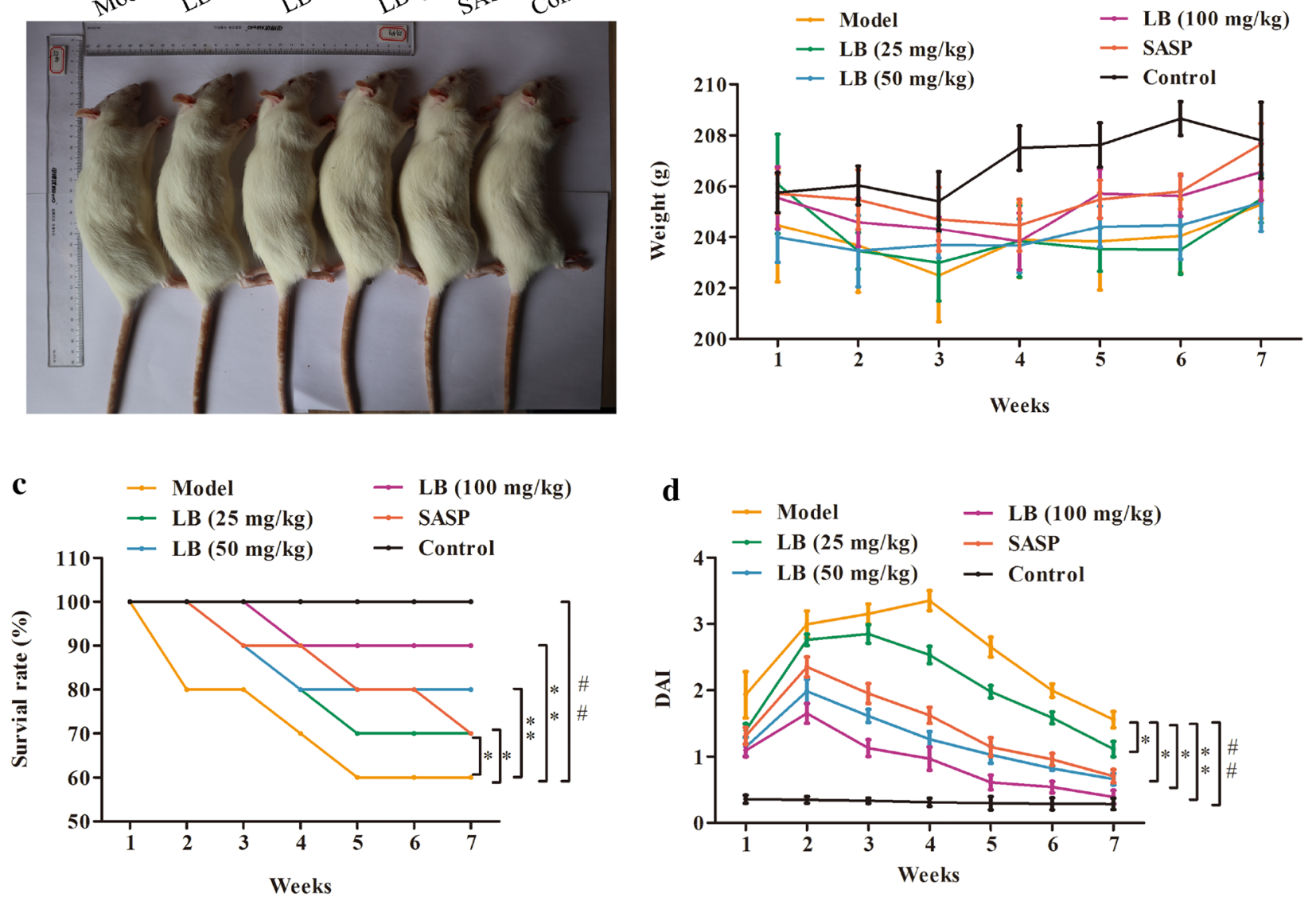

Fig. 1 Effect of LB on the survival rate and body weight in rats induced with TNBS. a, b The body weight loss of CD rats treated with LB was evaluated. $F=9.594, d f=41$. $\mathbf{c}$ The survival time of $C D$ rats treated with $L B$ was measured. $F=5.604, d f=41$. $\mathbf{d}$ The DAl score of rats from different groups was counted. $\mathrm{F}=15.32, \mathrm{df}=41$. The results were expressed as the mean $\pm \mathrm{SD}$ of at least three experiments. ${ }^{\#} P<0.01$ compared with the control group. ${ }^{*} P<0.05,{ }^{*} P<0.01$ compared with the model group

Effect of LB on the expression of INOS, MPO, SIgA and MDA in rats induced with TNBS

To further explore the role of LB in the expression of INOS, MPO, SIgA and MDA related to the infiltration of neutrophils in inflammatory tissues [33], we performed ELISA assay to detect the expression of INOS, MPO, SIgA and MDA in colonic tissues. As shown in Fig. 4, the expression of INOS, MPO and MDA was up-regulated and the expression of SIgA was down-regulated in TNBS-induced group compared to that in the control group, while treatment with LB led to a decrease in the expression of INOS, MPO and MDA and an increase of SIgA in colonic tissues.

\section{Effect of LB on epithelial-mesenchymal transition (EMT) in rats induced with TNBS}

Intestinal fibrosis is thought to be a common phenomenon in inflammatory bowel disease, and often causes serious complications in $\mathrm{CD}$, eventually leading to intestinal stenosis [34]. Masson's trichrome staining assay was performed to investigate the fibrotic changes histopathologically. The results of Fig. 5a demonstrated that TNBS could promote the inflammatory infiltration and fibrotic tissue accumulation, while LB treatment could reverse the established fibrosis and impeded the accumulation infiltration. Additionally, EMT has been found to be an important pathway in the progress of intestinal fibrosis recently. Western blotting assay was carried out to examine the expression levels of EMT-related proteins, including Slug, Snai, N-cadherin, E-cadherin and Vimentin. As shown in Fig. 5b, the expression levels of Snail, $\mathrm{N}$-cadherin and Vimentin were obviously over-expressed, and the expression levels of E-cadherin was notably low-expressed in rat induced with TNBS. After treatment with LB, the expression levels of Snail, N-cadherin and Vimentin were significantly down-regulated and the 

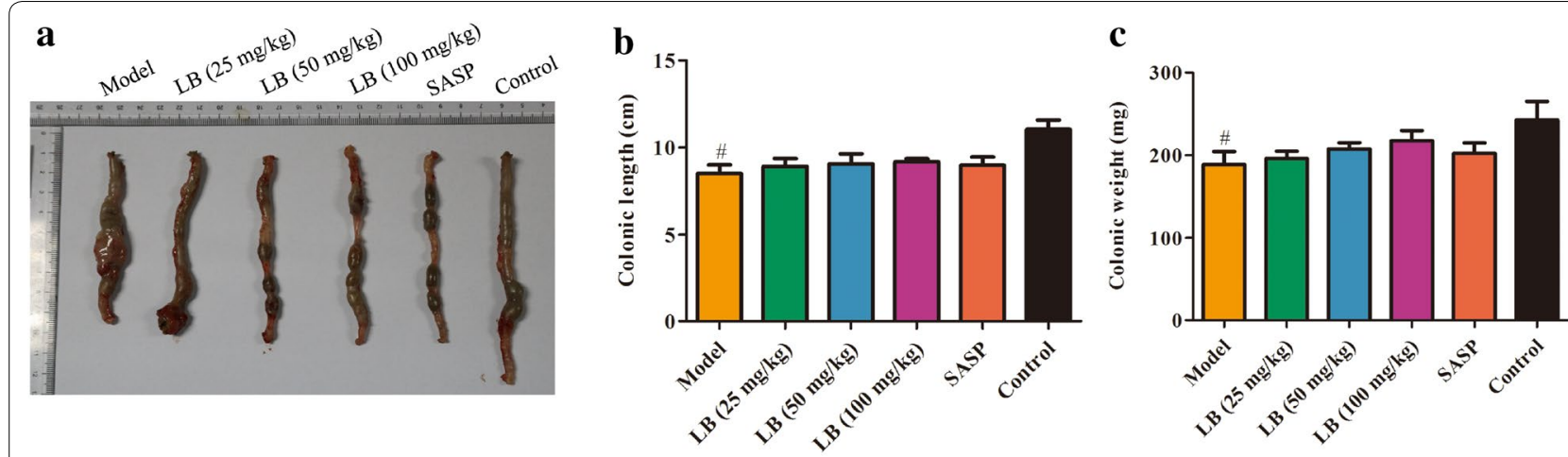

d

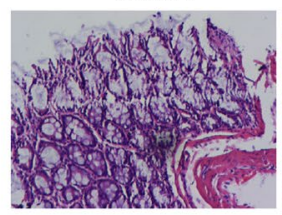

LB (100 mg/kg)

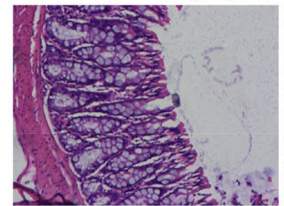

LB (25 mg/kg)

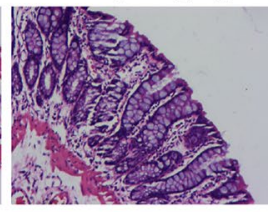

SASP

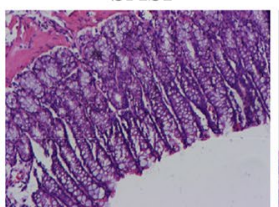

LB $(50$ mg/kg)

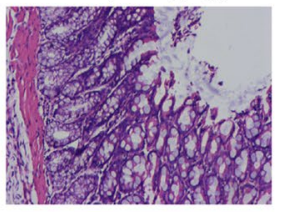

Control

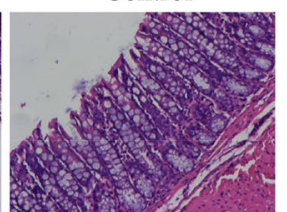

$\mathbf{e}$

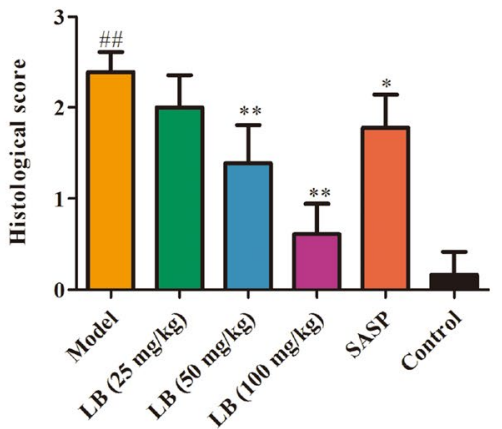

Fig. 2 Effect of $L B$ on the colonic length in rats induced with TNBS. a-c The colonic length $(F=10.79, d f=17)$ and weight $(F=5.472, d f=17)$ of $C D$ rats treated with $L B$ was measured. $\mathbf{d}$, e The severity of colonic inflammation of $C D$ rats treated with $L B$ was evaluated by $H \& E$ staining. $F=59.95$, $\mathrm{df}=53$. The results were expressed as the mean $\pm \mathrm{SD}$ of at least three experiments. ${ }^{\#} P<0.05,{ }^{\# \#} P<0.01$ compared with the control group. ${ }^{*} P<0.05$, ${ }^{*} P<0.01$ compared with the model group

expression level of E-cadherin was remarkably up-regulated in a dose depend manner. However, there were no significant changes in the expression of Slug. Besides, immunohistochemistry assay was performed to evaluate the expression levels of these proteins. The trend of the results was consistent with the trend of western blotting assay (Fig. 5c).

\section{Effect of LB on apoptosis in rats induced with TNBS}

Apoptosis is a programmed cell death occurring in various inflammatory diseases including CD [35]. Firstly, TUNEL assay was performed to evaluate the apoptotic degree of colonic tissues. As shown in Fig. 6a, TNBS could obviously promote the colonic tissue apoptosis, while treatment with LB had better improving effect on the apoptosis induced by TNBS in a dose dependent manner. Further, western blotting and immunohistochemistry assays were adopted to examine the expression levels of proteins related to apoptosis. The results of Fig. 6b, c demonstrated that TNBS could promote the expression levels of Bax, Cleaved-caspase- 3 and Cleavedcaspase-9, and suppress the expression of Bcl-2. Interestingly, LB treatment could reverse the effect of TNBS.

\section{Effect of LB on IL-6/STAT3/NF-KB signaling pathway in rats} induced with TNBS

IL-6/STAT3/NF-kB signaling pathway plays an important role in the development and progression of $C D$ [36]. The functional activity of NF- $\mathrm{\kappa B}$ was accessed by electrophoretic mobility shift assay (EMSA) (Fig. 7a). In this study, we further investigated the effect of $L B$ on the expression of IL-6/STAT3/NF-kB in CD rats by using qRT-PCR and western blot assays. As shown in Fig. $7 b, c$, the abundances of IL-6, p-STAT3, p-NF-kB (p65) were greatly increased and $\mathrm{p}-\mathrm{IKB}-\alpha$ was decreased in model group compared with control group. However, it was observed that SASP treatment lowered the expression of IL-6, p-STAT3, p-NF-kB (p65) and raised the expression of $\mathrm{p}-\mathrm{IKB}-\alpha$ as compared with the model group. Moreover, LB treatment had better inhibitory effects on the expression of IL-6/STAT3/NF-kB signaling pathway.

\section{Discussion}

CD is a transmural, segmental, asymmetric inflammatory bowel disease that affects any part of the digestive tract $[1,2]$. This disease is more common in European and American countries, but recently the incidence of 

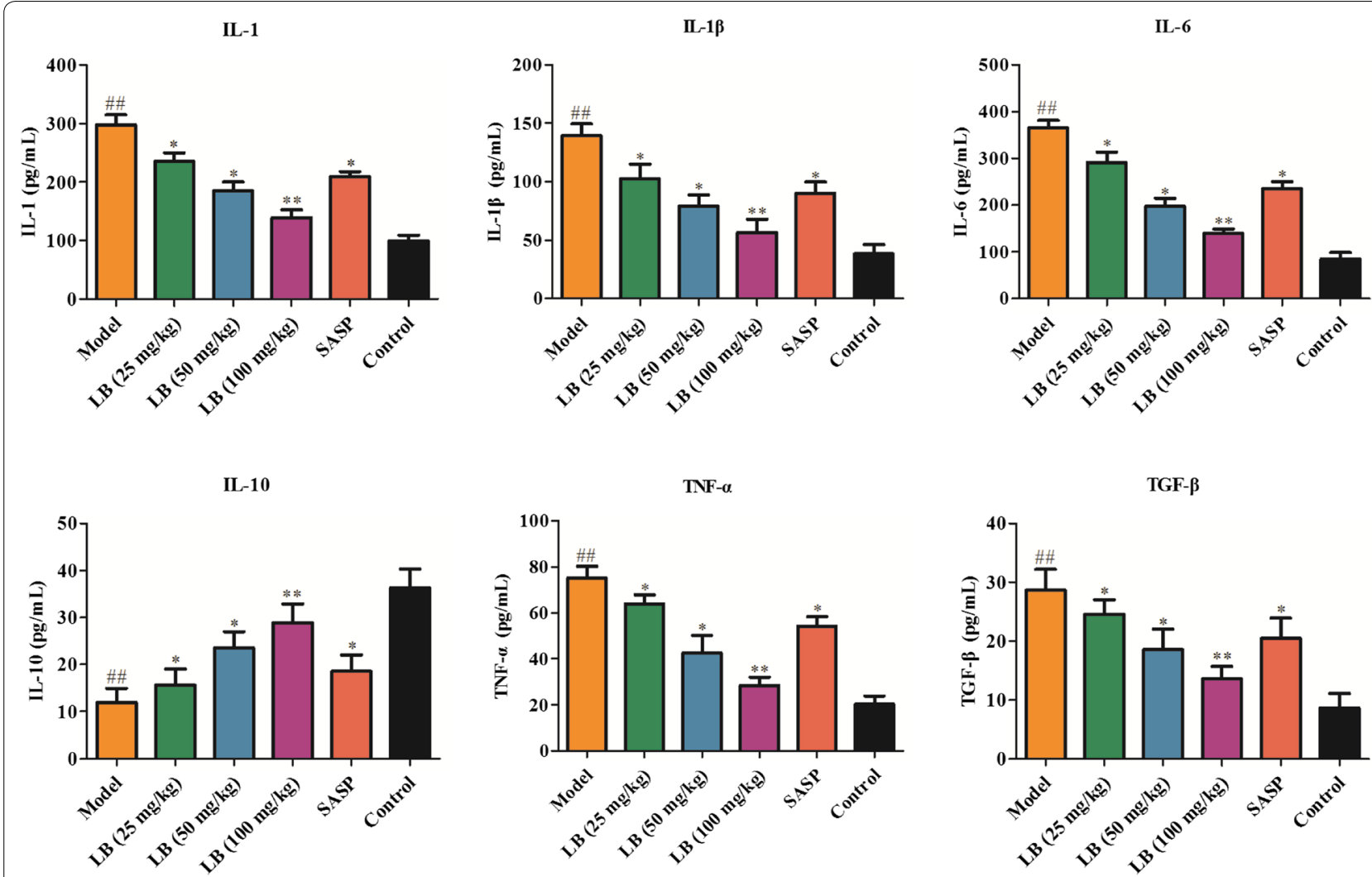

Fig. 3 Effect of $L B$ on the production of inflammatory cytokines in rats induced with TNBS. ELISA assay was performed to detect the production of cytokines, including IL-1 $(F=78.32, d f=17), I L-1 \beta(F=35.24, d f=17), I L-6(F=119.5, d f=17), T N F-a(F=57.35, d f=17), I L-10(F=18.79, d f=17)$ and TGF- $\beta(F=17.67, d f=17)$ in colonic tissues of $C D$ rats from different groups. The results were expressed as the mean $\pm S D$ of at least three experiments. ${ }^{\# \#} P<0.01$ compared with the control group. ${ }^{*} P<0.05$, ${ }^{* *} P<0.01$ compared with the model group

CD is increasing obviously in China, and the incidence of CD between 2004 and 2008 is 8.5 times higher than that between 1989 and 1993 in China [37, 38]. In addition, the etiology and pathogenesis of $C D$ are not yet fully understood. At present, it is believed that CD may be caused by mucosal damage caused by intestinal immune hyperactivity due to the infection, diet and other environmental factors. Immune abnormalities have been recognized to play a very important role in the pathogenesis of $\mathrm{CD}$, including inflammatory transmitters, cytokines and immune regulation [39-41].

Nowadays, clinical treatment of $\mathrm{CD}$ is mainly based on drugs according to the severity of $\mathrm{CD}$, and commonly used drugs are aminosalicylic acid, glucocorticoids, immunosuppressors and anti TNF- $\alpha$ biological agents [42]. Actually, 5-aminosalicylic acid (5-ASA) can not effectively alleviate $C D$, the curative effect is uncertain, and has many side effects [43]. Clinical studies have shown that the abuse of glucocorticoids and immunosuppressors not only can not control the degree of CD, but also cause seriously drug-related complications, leading to serious damage to the body [44]. As the most advanced medical method for $\mathrm{CD}$ at present, the treatment efficiency of the combination of anti-tumor necrosis factor and mercaptopurine is only about $50 \%[45,46]$. Therefore, most of the drugs and treatments currently used in the treatment of CD clinically have poor efficacy and side effects.

Over the years, Chinese medicine has accumulated a wealth of experience in the treatment of $\mathrm{CD}$, a large number of literature reports and modern research have confirmed that Chinese medicine in the treatment of $C D$ has a significant effect [47-49]. LB as a Chinese herb has been commonly used for the treatment of coronary heart disease, angina, and acute myocardial infarction, and the ethyl acetate of LB can promote inflammatory response induced with LPS through inhibiting ROS production in vascular smooth muscle cells and macrophages [19-22]. However, the role of LB on CD and the underlying mechanisms remain unknown. In the present study, we found that LB could prolong the survival time of CD rats, ameliorate TNBS-induced weight loss, colonic length and inflammatory infiltration. 
iNOS

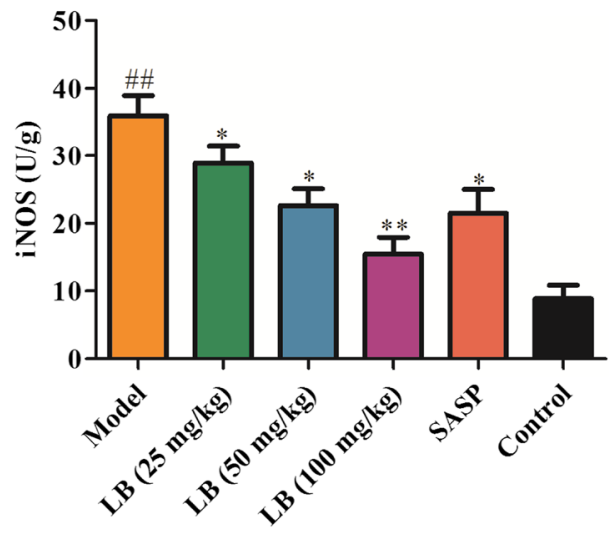

$\operatorname{SIg} \mathbf{A}$

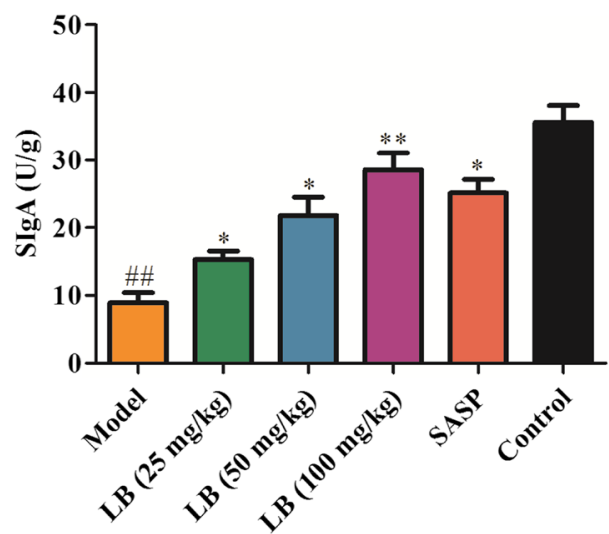

MPO

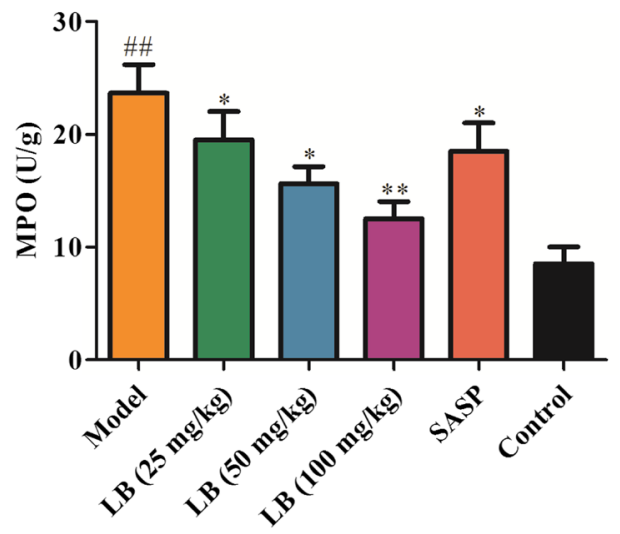

MDA

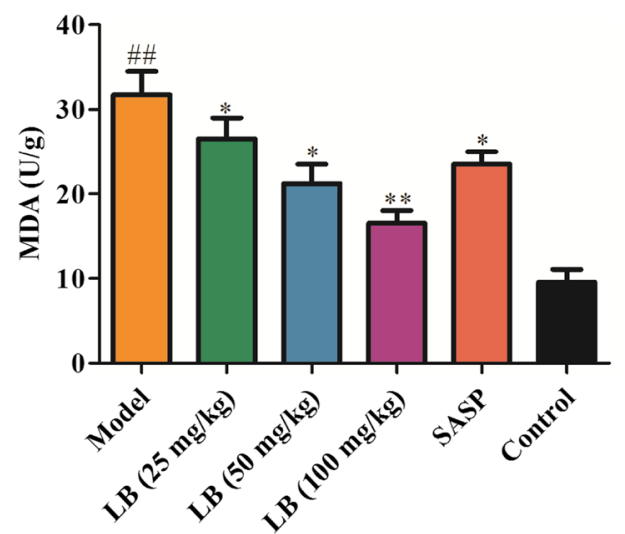

Fig. 4 Effect of LB on the expression of INOS, MPO, SIgA and MDA in rats induced with TNBS. ELISA assay was performed to detect the production of INOS $(F=36.97, d f=17), M P O(F=20.34, d f=17)$, SlgA $(F=58.30, d f=17)$ and MDA $(F=42.37, d f=17)$ in colonic tissues of $C D$ rats from different groups. The results were expressed as the mean \pm SD of at least three experiments. ${ }^{\# \#} P<0.01$ compared with the control group. ${ }^{*} P<0.05$, ${ }^{* *} P<0.01$ compared with the model group

Recent studies have shown that CD is a typical type Th I reaction. In addition to immune cells, non-immune cells of intestinal mucosa, such as epithelial cells, vascular endothelial cells and interstitial cells, also participate in the immune response and inflammatory process of CD through releasing various cytokines and inflammatory mediators, which can lead to the occurrence and development of CD. Further, regulating the expression of some cytokines can treat $C D[50,51]$. For example, high level of IL-6 is associated with active CD [52]. Nunberg et al. unveiled impaired IL-10 receptor-mediated suppression in monocyte from patients with Crohn's disease [53]. In this study, we found that the administration of LB in rats induced with TNBS led to a significant decrease of the production of IL- 1, IL- $1 \beta$, IL- 6 , TGF- $\beta$ and TNF- $\alpha$, and an obvious increase of secretion of IL-10. Thereafter, we further measured the concentrations of some cytokines related to inflammation in $\mathrm{CD}$, including INOS, MPO,
SIgA and MDA. Previous literatures illuminated that the positive correlations of INOS, MPO and MDA with the severity of CD [54-56]. However, SIgA was lowly expressed in CD patients and SIgA might be involved in CD pathogenesis [57]. Our findings expounded that LB treatment ameliorated the expression of INOS, MPO and MDA in TNBS-induced rats, while the activity of SIgA was significantly inhibited by LB. These results indicated that LB had a positive role in the modulation of cytokine production in the setting of CD.

Chronic inflammation and injury of the intestine can lead to serious complications such as intestinal fibrosis and intestinal obstruction. Therefore, the research on the pathogenesis of $C D$ intestinal fibrosis has become a popular direction [58]. Under normal physiological conditions, fibroblasts aggregate in damaged tissues to promote the repair of damaged tissues. The ability of fibroblasts in the intestinal mucosa to aggregate into 


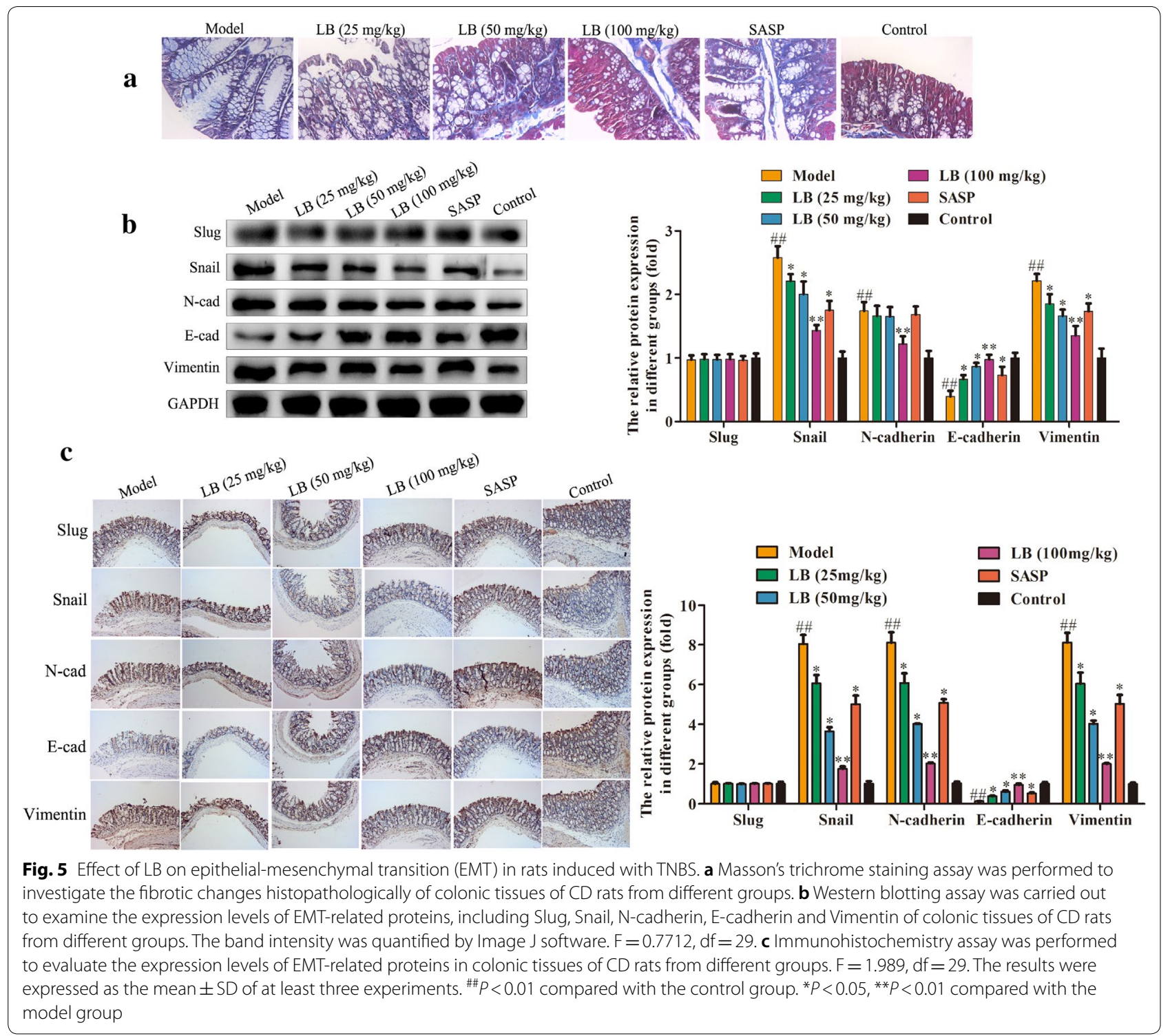

damaged tissues in patients with $C D$ is weakened and the wound healing function is disordered compared with normal people. When the intestine is damaged, the epithelial cells of the intestinal mucosa can migrate under the induction of many growth factors. When the fibroblasts in the intestinal tract can not repair the defect of mucosal tissues, intestinal epithelial cells can be transformed into interstitial cells, and migrate to the damaged area, so that the defective intestinal barrier can be repaired [59]. Intestinal epithelial cells may undergo EMT or apoptosis in the presence of persistent adverse environmental effects. In fact, the cytokine TGF- $\beta$, which plays an important role in the development of EMT, can promote the apoptosis of epithelial cells [60]. Exposure to TGF- $\beta$ can induce apoptosis of a large number of epithelial cells. In addition, the weakening of adhesion between cells and basement membrane during EMT is also an important pathway of apoptosis [61]. Therefore, if EMT persists and fibroblast apoptosis is inhibited, it will lead to progressive development of intestinal fibrosis. In the present study, we found that LB treatment could reverse the established fibrosis and impeded the accumulation infiltration, and after treatment with LB, the expression levels of Snail, N-cadherin and Vimentin were significantly down-regulated and the expression level of E-cadherin was remarkably up-regulated in a dose depend manner. Further, treatment with LB had better improving effect on the apoptosis induced with TNBS in a dose dependent manner. 


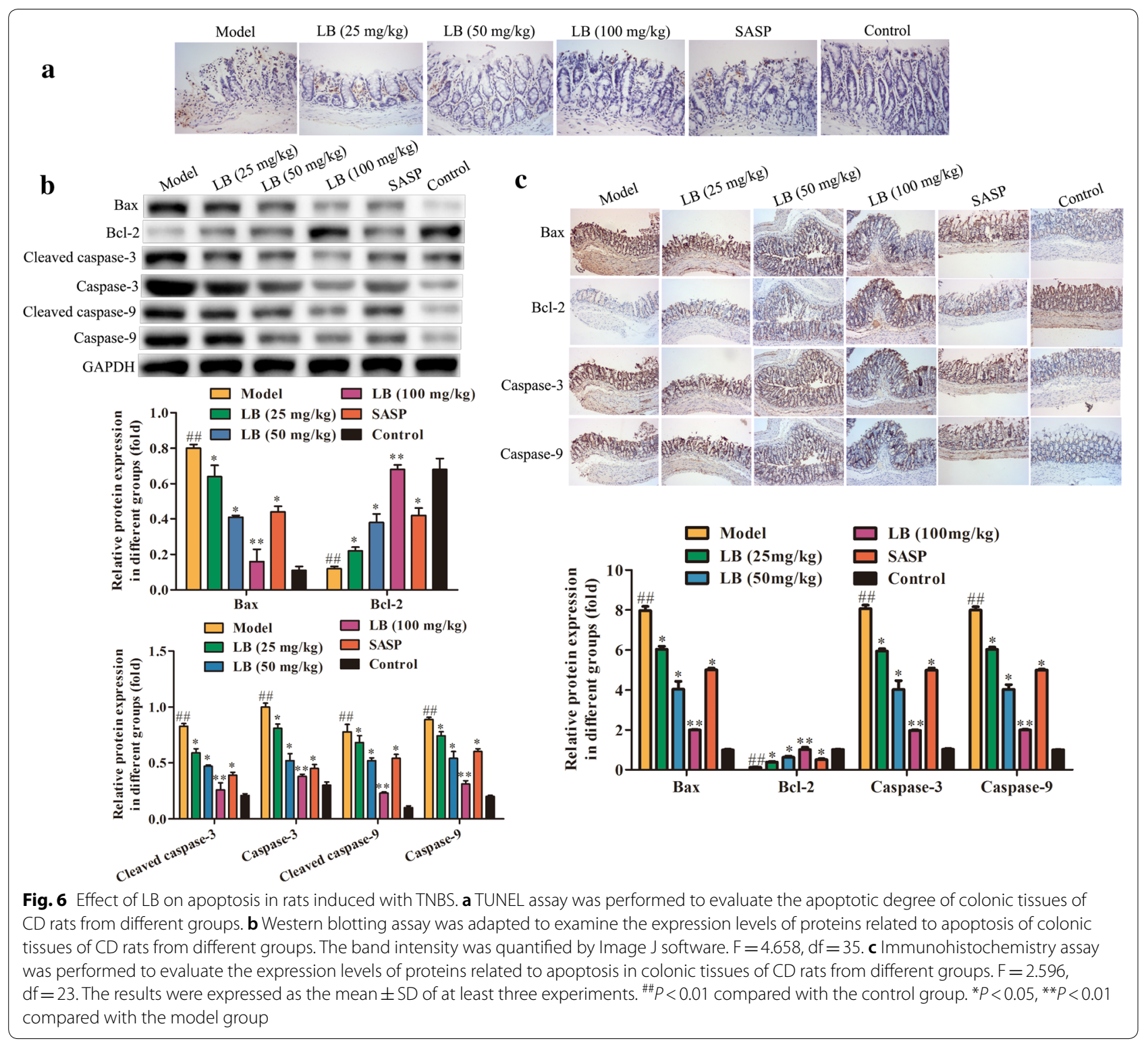

IL-6/STAT3 signaling pathway plays an important role in the occurrence and development of IBD [62]. The activation of IL-6/STAT3 signal transduction pathway mainly depends on the recognition and binding of IL- 6 and sIL-6R on the target cell surface to form sIL-6R/IL-6 complex, which further activates gp130 on the surface of cell membrane. Gp130 is stimulated to form homologous dimers, which activates JAK associated with gp130 and receptor tyrosine kinase, and binds to STAT3 protein. This kinase cascade phosphorylates STAT3 and actives NF- $\mathrm{kB}$ signaling pathway, thereby regulating expression of inflammatory cytokines [63].
In this study, we found that LB could inhibit the activation of IL-6/STAT3/NF-kB signaling pathway.

\section{Conclusions}

To conclude, our study suggested that LB could be beneficial regarding ameliorating the damage to colon length, suppressing inflammatory cytokines and inhibiting apoptosis through regulation of IL-6/STAT3/NF-kB signaling pathway, which may represent a novel approach to treat $\mathrm{CD}$ and provide alternative choice for disorders associated with $\mathrm{CD}$. 

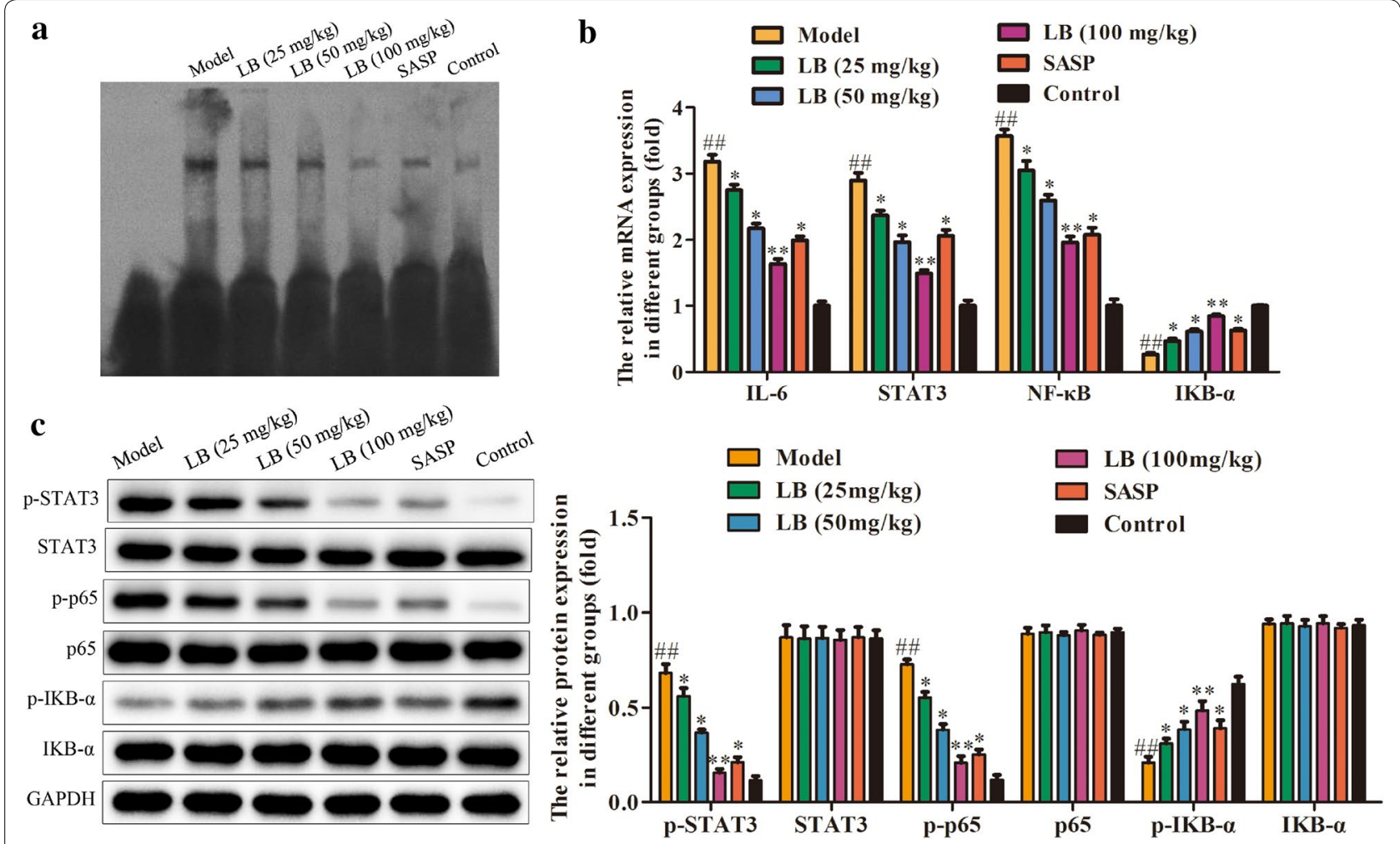

Fig. 7 Effect of LB on IL-6/STAT3/NF-KB signaling pathway in rats induced with TNBS. a The functional activity of NF-KB was accessed by EMSA. $\mathbf{b}$, $\mathbf{c}$ qRT-PCR and western blot assays were performed to evaluate the mRNA and protein expression of IL-6/STAT3/NF-KB signaling pathway of colonic tissues of $C D$ rats from different groups. The band intensity was quantified by Image J software. The results were expressed as the mean \pm SD of at least three experiments. ${ }^{\# \#} P<0.01$ compared with the control group. ${ }^{*} P<0.05,{ }^{* *} P<0.01$ compared with the model group

\section{Abbreviations}

CD: Crohn's disease; RD: Resina Donis; LB: Loureirin B; TNBS: 2,4,6-trinitrobenzenesulfonic acid solution; PVDF: polyvinylidene difluoride membrane.

\section{Acknowledgements}

This work was supported by Suzhou Municipal Science and Technology Bureau (No. SYSD2017126).

\section{Authors' contributions}

$X S$, XW conceived the manuscript; KW, ZX, ZH performed the experiments; $\mathrm{BW}, \mathrm{XY}$ analysed the results and provided materials and resources. All authors read and approved the final manuscript.

\section{Funding}

Suzhou Municipal Science and Technology Bureau (No. SYSD2017126).

\section{Availability of data and materials}

The datasets used during the present study are available from the corresponding author upon reasonable request.

\section{Ethics approval and consent to participate}

Experiments were approved by Animal Experimentation Ethics Committee of the Nanjing Medical University. Consent to participate is not relevant to this manuscript.

\section{Consent for publication}

Not relevant to this manuscript.

\section{Competing interests}

The authors declare that they have no competing interests.
Received: 25 July 2019 Accepted: 27 December 2019

Published online: 06 January 2020

\section{References}

1. Carter D, Lang A, Eliakim R. Endoscopy in inflammatory bowel disease. Minerva Gastroenterol Dietol. 2013;59(3):273-84.

2. Rieder F, Bettenworth $\mathrm{D}, \mathrm{Ma} \mathrm{C}$, et al. An expert consensus to standardise definitions, diagnosis and treatment targets for anti-fibrotic stricture therapies in Crohn's disease. Aliment Pharmacol Ther. 2018;48(3):347-57.

3. Ren J, Tao Q, Wang X, et al. Efficacy of T2 in active Crohn's disease: a prospective study report. Dig Dis Sci. 2007;52(8):1790-7.

4. Wei X, Gong J, Zhu J, et al. Therapeutic effects of triptolide on interleukin-10 gene-deficient mice with colitis. Int Immunopharmacol. 2008:8(13-14):1808-12.

5. Han Y, Lin MB, He YG, et al. Laparoscopic surgery for inflammatory bowel disease - the experience in China. J Invest Surg. 2013;26(4):180-5.

6. Peng $\mathrm{QH}$, Wang $\mathrm{YF}$, He MQ, et al. Clinical literature review of 1858 Crohn's disease cases requiring surgery in China. World I Gastroenterol. 2015;21(15):4735-43.

7. Baumgart DC, Carding SR. Inflamatory bowel disease: cause and immunobiology. Lancet. 2007:369(9573):1627-40.

8. Baumgart DC, Sandborn WJ. Inflammatory bowel disease: clinical aspects and established and evolving therapies. Lancet. 2007:369(9573):1641-57.

9. Ko JK, Auyeung KK. Inflammatory bowel disease: etiology, pathogenesis and current therapy. Curr Pharm Des. 2014:20(7):1082-96.

10. Mudter J, Neurath MF. Apoptosis of T cells and the control of inflammatory bowel disease: therapeutic implications. Gut. 2007;56(2):293-303. 
11. Atreya R, Zimmer M, Bartsch B, et al. Antibodies against tumor necrosis factor (TNF) induce T-cell apoptosis in patients with inflammatory bowel diseases via TNF receptor 2 and intestinal CD14 ${ }^{+}$macrophages. Gastroenterology. 2011;141(6):2026-38.

12. Musso A, Dentelli $P$, Carlino A, et al. Signaling transducers and activators of transcription 3 signaling pathway: an essential mediator of inflammatory bowel disease and other forms of intestinal inflammation. Inflamm Bowel Dis. 2005;11(2):91-8.

13. Gross V, Andus T, Caesar I, et al. Evidence for continuous stimulation of interleukin-6 production in Crohn's disease. Gastroenterology. 1992;102(2):514-9.

14. Atreya R, Mudter J, Finotto S, et al. Blockade of interleukin 6 trans signaling suppresses T-cell resistance against apoptosis in chronic intestinal inflammation: evidence in crohn disease and experimental colitis in vivo. Nat Med. 2000;6(5):583-8.

15. Yamamoto $M$, Yoshizaki K, Kishimoto T, et al. IL-6 is required for the development of Th1 cell-mediated murine colitis. J Immunol. 2000;164(9):4878-82.

16. Nishihara M, Ogura H, Ueda N, et al. IL-6-gp130-STAT3 in T cells directs the development of $\mathrm{IL}-17^{+}$Th with a minimum effect on that of Treg in the steady state. Int Immunol. 2007;19(6):695-702.

17. Mudter J, Neurath MF. IL-6 signaling in inflammatory bowel disease: pathophysiological role and clinical relevance. Inflamm Bowel Dis. 2007;13(8):1016-23.

18. Atreya I, Atreya R, Neurath MF. NF-kappaB in inflammatory bowel disease. J Intern Med. 2008;263(6):591-6.

19. Xu J, Xiong T, Yang Y, et al. Resina Draconis as a topical treatment for pressure ulcers: a systematic review and meta-analysis. Wound Repair Regen. 2015;23(4):565-74.

20. Liu H, Lin S, Xiao D, et al. Evaluation of the wound healing potential of Resina Draconis (Dracaena cochinchinensis) in animal models. Evid Based Complement Alternat Med. 2013;2013:709865.

21. Heo SK, Yi HS, Yun HJ, et al. Ethylacetate extract from Draconis Resina inhibits LPS-induced inflammatory responses in vascular smooth muscle cells and macrophages via suppression of ROS production. Food Chem Toxicol. 2010;48(5):1129-36.

22. Lu H, Teng J, Wu H. A review on the study of Resina Draconis produced in China. Zhong Yao Cai. 2003;26(6):459-61.

23. Jian-Peng $H$, Rong $Z$, Min T, et al. Loureirin B inhibits the proliferation of hepatic stellate cells and the Wnt/ $\beta$-catenin signaling pathway by regulating miR-148-3p. Cell Mol Biol Lett. 2018;23(1):35.

24. Yin $\mathrm{S}, \mathrm{Hu} \mathrm{Q}$, Luo J, et al. Loureirin $\mathrm{B}$, an essential component of Sanguis Draxonis, inhibits Kv1.3 channel and suppresses cytokine release from Jurkat T cells. Cell Biosci. 2014;4(1):78.

25. Bai X, He T, Liu J, et al. Loureirin B inhibits fibroblast proliferation and extracellular matrix deposition in hypertrophic scar via TGF- $\beta / S m a d$ pathway. Exp Dermatol. 2015;24(5):355-60.

26. Yu J, Guangping Z, Dong Y, et al. Bioactivity-guided fractionation of the traditional Chinese medicine Resina Draconis reveals Loureirin B as a PAl-1 inhibitor. Evid Based Complement Altern Med. 2017;2017:1-8.

27. Tomasello $G$, Sinagra E, Raimondo $D$, et al. Validation of a modified model of TNBS-induced colitis in rats. How to induce a chemical colitis in rats. Acta Biomed. 2015;86(1):92-6.

28. Yang XS, Zhang L, Shi XY, Zhang YL, Lv YM. Current diagnostic status, clinical and pathologic manifestation for a Crohn's disease. Beijing Da Xue Xue Bao Yi Xue Ban. 2006;38(4):407-10.

29. Bao $\mathrm{CH}, \mathrm{Wu} \mathrm{LY}$, et al. Moxibustion down-regulates colonic epithelial cell apoptosis and repairs tight junctions in rats with Crohn's disease. World J Gastroenterol. 2011;17(45):4960.

30. Yao J, Lu Y, Zhi M, et al. Dietary n-3 polyunsaturated fatty acids ameliorate Crohn's disease in rats by modulating the expression of PPAR- $\gamma /$ NFAT. Mol Med Rep. 2017:16(6):8315-22.

31. Cooper HS, Murthy SN, Shah RS, et al. Clinicopathologic study of dextran sulfate sodium experimental murine colitis. Lab Invest. 1993;69(2):238-49.

32. Rogler G, Andus T. Cytokines in inflammatory bowel disease. World J Surg. 1998:22(4):382-9.

33. Tung CC, Wong JM, Lee WC, et al. Combining TNFSF15 and ASCA IgA can be used as a predictor for the stenosis/perforating phenotype of Crohn's disease. J Gastroenterol Hepatol. 2014;29(4):723-9.
34. Zorzi F, Calabrese E, Monteleone G. Pathogenic aspects and therapeutic avenues of intestinal fibrosis in Crohn's disease. Clin Sci. 2015:129(12):1107-13.

35. Poniedziałek B, Rzymski P, Karczewski J. Increased apoptosis of regulatory T cells in Crohn's disease. Hepatogastroenterology. 2014;61(130):382-4.

36. Takac B, Mihaljević S, Stefanić M, et al. Importance of interleukin-6 in pathogenesis of inflammatory bowel disease. Coll Antropol. 2014:38(2):659-64.

37. Wang YF, Ouyang Q, Hu RW. Progression of inflammatory bowel disease in China. J Dig Dis. 2010;11(2):76-82.

38. Zheng JJ, Zhu XS, Huangfu Z, et al. Prevalence and incidence rates of Crohn's disease in mainland China: a meta-analysis of 55 years of research. J Dig Dis. 2010;11(3):161-6.

39. Palmela C, Torres J, Cravo M. New trends in inflammatory bowel disease. GE Port J Gastroenterol. 2015;22(3):103-11.

40. Fernandes C, Allocca M, Danese $S$, et al. Progress with anti-tumor necrosis factor therapeutics for the treatment of inflammatory bowel disease. Immunotherapy. 2015;7(2):175-90.

41. Church PC, Turner D, Feldman BM, et al. Systematic review with metaanalysis: magnetic resonance enterography signs for the detection of inflammation and intestinal damage in Crohn's disease. Aliment Pharmacol Ther. 2015;41(2):153-66.

42. Cury DB, Moss AC. Treatment of Crohn's disease in pregnant women: drug and multidisciplinary approaches. World J Gastroenterol. 2014;20(27):8790-5.

43. Sanapalli BKR, Kannan E, Balasubramanian S, et al. Pluronic lecithin organogel of 5-aminosalicylic acid for wound healing. Drug Dev Ind Pharm. 2018;2:1-9.

44. Renna S, Orlando A, Cottone M. Comparing medical treatments for Crohn's disease. J Comp Eff Res. 2013;2(2):135-49.

45. Desai RJ, Gagne JJ, Lii J, et al. Comparative risk of incident venous thromboembolism in patients with inflammatory bowel disease initiating tumour necrosis factor-a inhibitors or non biologic agents: a cohort study. CMAJ. 2017:189(47):E1438-47.

46. Walters TD, Kim MO, Denson LA, et al. Increased effectiveness of early therapy with anti-tumor necrosis factor-a vs an immunomodulator in children with Crohn's disease. Gastroenterology. 2014;146(2):383-91.

47. Liu C, Dunkin D, Lai J, et al. Anti-inflammatory effects of Ganoderma lucidum triterpenoid in human Crohn's disease associated with downregulation of NF-KB signaling. Inflamm Bowel Dis. 2015;21(8):1918-25.

48. Laverny G, Penna G, Vetrano S, et al. Efficacy of a potent and safe vitamin $D$ receptor agonist for the treatment of inflammatory bowel disease. Immunol Lett. 2010;131(1):49-58.

49. Mencarelli A, Renga B, Palladino G, et al. The plant sterol guggulsterone attenuates inflammation and immune dysfunction in murine models of inflammatory bowel disease. Biochem Pharmacol. 2009;78(9):1214-23.

50. Kontoyiannis D, Pasparakis M, Pizarro TT, et al. Impaired on/off regulation of TNF biosynthesis in mice lacking TNF AU-rich elements: implications for joint and gut-associated immunopathologies. Immunity. 1999;10(3):387-98.

51. Schreiber S, Nikolaus S, Hampe J, et al. Tumour necrosis factor alpha and interleukin 1 beta in relapse of Crohn's disease. Lancet. 1999:353(9151):459-61.

52. Ito H. IL-6 and Crohn's disease. Curr Drug Targets Inflamm Allergy. 2003;2(2):125-30.

53. Nunberg MY, Werner L, Kopylov U, et al. Impaired IL-10 receptor-mediated suppression in monocyte from patients with Crohn disease. J Pediatr Gastroenterol Nutr. 2018;66(5):779-84

54. Leeb SN, Vogl D, Gunckel M, et al. Reduced migration of fibroblasts in inflammatory bowel disease: role of inflammatory mediators and focal adhesion kianse. Gastroenterology. 2003;125(5):1341-54.

55. Tarnawski AS. Cellular and molecular mechanisms of gastrointestinal ulcer healing. Dig Dis Sci. 2005;50(Suppl 1):S24-33.

56. Soufli I, Toumi R, Rafa H, Touil-Boukoffa C. Overview of cytokines and nitric oxide involvement in immuno-pathogenesis of inflammatory bowel diseases. World J Gastrointest Pharmacol Ther. 2016;7(3):353-60.

57. Luo X, Yu Z, Deng C, et al. Baicalein ameliorates TNBS-induced colitis by suppressing TLR4/MyD88 signaling cascade and NLRP3 inflammasome activation in mice. Sci Rep. 2017:7(1):16374. 
58. Szczeklik K, Krzyściak W, Cibor D, et al. Markers of lipid peroxidation and antioxidant status in the serum and saliva of patients with active Crohn disease. Pol Arch Intern Med. 2018;128(6):362-70.

59. Marteau P, Colombel JF, Nemeth J, Vaerman JP, Dive JC, Rambaud JC. Immunological study of histologically non-involved jejunum during Crohn's disease: evidence for reduced in vivo secretion of secretory lgA. Clin Exp Immunol. 1990:80(2):196-201.

60. Song J. EMT or apoptosis: a decision for TGF-beta. Cell Res. 2007;17(4):289-90.

61. Zhang HY, Phan SH. Inhibition of myofibroblast apoptosis by transforming growth factor beta. Am J Respir Cell Mol Biol. 1999;21(6):658-65.
62. Waldner MJ, Neurath MF. Master regulator of intestinal disease: IL-6 in chronic inflammation and cancer development. Semin Immunol. 2014:26(1):75-9.

63. Pitman H, Innes BA, Robson SC, et al. Altered expression of interleukin-6, interleukin-8 and their receptors in decidua of women with sporadic miscarriage. Hum Reprod. 2013;28(8):2075-86.

\section{Publisher's Note}

Springer Nature remains neutral with regard to jurisdictional claims in published maps and institutional affiliations.
Ready to submit your research? Choose BMC and benefit from:

- fast, convenient online submission

- thorough peer review by experienced researchers in your field

- rapid publication on acceptance

- support for research data, including large and complex data types

- gold Open Access which fosters wider collaboration and increased citations

- maximum visibility for your research: over 100M website views per year

At BMC, research is always in progress.

Learn more biomedcentral.com/submissions 